\title{
Aufsatz
}

Heinrich Brauß* und Joachim Krause

\section{Was will Russland mit den vielen Mittelstreckenwaffen?}

https://doi.org/10.1515/sirius-2019-2005

Zusammenfassung: Der Aufsatz befasst sich mit der Frage, was Russland dazu veranlasst hat, nach langer Pause erneut in nuklearfähige Mittelstreckenwaffen zu investieren, die vornehmlich gegen Ziele in Europa gerichtet sind. Die Autoren gelangen zu dem Ergebnis, dass die russische Rüstung nicht mit dem Aktions-Gegenreaktions-Theorem erklärt werden kann, sondern sich aus dem spezifisch russischen Militäransatz ergibt. Russland verspricht sich offenkundig entscheidende strategische Vorteile im Sinne einer Eskalationsdominanz gegenüber den USA und der NATO für den Fall eines regionalen Krieges in Europa eines Krieges, der aller Voraussicht nach nur von Russland ausgehen würde. Die westliche Allianz sollte auf die Politik Moskaus vor allem mit einer Verbesserung der Fähigkeit zur konventionellen Verteidigung der baltischen Staaten und Polens reagieren, denn die russische Mittelstreckenrüstung macht nur Sinn im Rahmen eines regionalen Kriegsszenarios. Allerdings wird die NATO nicht umhinkommen, das eigene Nukleardispositiv entsprechend anzupassen.

Schlüsselbegriffe: NATO, Russland, Mittelstreckenraketen, INF-Vertrag, Ukraine, baltische Staaten, Verteidigungsstrategie

\footnotetext{
Abstract: The article asks why Russia has started again to invest in intermediate-range missiles, which can be used both in conventional and nuclear roles. The authors arrive at the conclusion that the Russian efforts cannot be explained by resorting to the action-reaction scheme. Rather, the nature of the specific Russian approach towards military issues should be taken into consideration. Russia obviously tries to achieve major strategic

*Kontakt: Generalleutnant a. D. Heinrich Brauß, Senior Associate Fellow der Deutschen Gesellschaft für Auswärtige Politik, bis 2018 Beigeordneter Generalsekretär der NATO für Verteidigungspolitik und Streitkräfteplanung, E-Mail: brauss@af.dgap.org Prof. Dr. Joachim Krause: Geschäftsführender Herausgeber der Zeitschrift SIRIUS und Direktor des Instituts für Sicherheitspolitik an der Universität Kiel, E-Mail: jkrause@politik.uni-kiel.de.
}

advantages in case of a regional war taking place in Europe by having available a capability for escalation dominance. Any such war can only be initiated by Russia, not by NATO. The Western alliance should respond by improving the conventional defence capabilities with which it is going to protect the Baltic States and Poland. Besides that, NATO will have to undergo a review of its nuclear forces.

Keywords: NATO, Russia, Intermediate Nuclear Forces, INF-Treaty, Ukraine, Baltic States, Defence Strategy

\section{Einleitung}

Deutschland sorgt sich angesichts des Streits über die Einhaltung des Vertrags über das Verbot landgestützter nuklearer Mittelstreckenwaffen (INF) um die Gefahr eines neuen Rüstungswettlaufs. Immer neue Ideen werden entwickelt, die dazu beitragen sollen, dass es doch noch gelingt, zu einer wie auch immer gearteten Verständigung zu kommen - bislang ohne Erfolg.

Deutsche Außen- und Sicherheitspolitik ist heute primär von einem Ansatz gekennzeichnet, der auf Vermittlung und Besänftigung setzt und die eigene Sicherheitslage erst einmal zurückstellt. Im Prinzip ist an einem derartigen Ansatz nichts auszusetzen, wenn er zu den gewünschten Ergebnissen und zu einer effektiven Verbesserung der Sicherheit führt (und nicht zu problematischen Scheinlösungen). Sollte das nicht der Fall sein - und viel spricht dafür, dass es hier so ist - dann wäre es an der Zeit, strategische Logik zu bemühen und sich in aller Offenheit jener Frage zu stellen, die eigentlich an den Anfang der heutigen Debatte gehört hätte: Was hat die russische Seite dazu gebracht, nach mehr als 30 Jahren Pause in nuklearfähige Mittelstreckenwaffen zu investieren? Was können die strategischen und politischen Ziele sein, die die russische Führung damit verbindet? Was bedeutet das für unsere Sicherheit? Und was bleibt zu tun? 


\section{Das Ausmaß der russischen Auf- rüstung mit Mittelstreckenwaffen}

Am Anfang dieser Überlegungen steht ein Befund, der in der deutschen Debatte weitgehend ignoriert wird: Das Ausmaß und die Breite der russischen Rüstungsanstrengungen im Bereich der nuklearfähigen Mittelstreckenwaffen übersteigen alles bislang Befürchtete. Zum Großteil handelt es sich dabei um Marschflugkörper, die mit Hilfe von Terrainverfolgungsradar in niedriger Höhe operieren und mit hoher Präzision Ziele bekämpfen können. ${ }^{1}$ Nicht nur dass Russland unter dem Bruch des INF-Vertrags einen landgestützten Marschflugkörper (9M729, SSC-8 in der NATO-Klassifikation) ${ }^{2}$ entwickelt und getestet hat, es hat bereits an vier verschiedenen Orten des europäischen Russlands vier Bataillone mit insgesamt 64 Werfern stationiert. ${ }^{3}$ Zudem wurde vor wenigen Jahren ein seegestützter nuklearwaffenfähiger Marschflugkörper des Typs Kalibr 3M14 eingeführt, der eine Reichweite von über $2.000 \mathrm{~km}$ hat und der nicht nur von Überwasserschiffen, sondern auch von getauchten U-Booten der Kilo-Klasse und der neuen Jasen-Klasse unter Wasser gestartet werden kann (3M14 PL). Von den U-Booten der Jasen-Klasse befindet sich gegenwärtig eine Einheit auf See, weitere werden gerade eingeführt bzw. gebaut. Die russische Marine stattet derzeit eine ganze Reihe von Überwasserschiffen mit Kalibr 3M14 T/S-Flugkörpern aus, die bei Reichweiten von bis $\mathrm{zu} 2.600 \mathrm{~km}$ von unterschiedlichen Positionen aus Angriffe auf Landziele in Europa erlauben. Hinzu kommt, dass auch eine weitere Modernisierung luftgestützter Marschflugkörper stattgefunden hat, die radar-

1 Vgl. die tabellarische Übersicht im Anhang.

2 Zu den technischen Details der SSC-8 vgl. die Angaben auf der Webseite des CSIS „Missile Defense Project“ zum Thema „SSC-8 (Novator 9M729), https://missilethreat.csis.org/missile/ssc-8-novator-9m729/. 3 Bereits 2017 hatte die New York Times davon berichtet, dass Russland zwei Bataillone mit SSC-8 Marschflugkörpern stationiert habe, davon eines in Kapustin Jar, der andere Stationierungsort sei nicht bekannt, vgl. Michael Gordon: „Russia Deploys Missile, Violating Treaty and Challenging Trump“, New York Times, 14.2.2017. Diese Meldung wurde einen Monat später von dem stellvertretenden Vorsitzenden der Joint Chiefs of Staff, General Paul Selva, im Rahmen einer Kongressanhörung bestätigt; vgl. Michael Gordon: „Russia Has Deployed Missile Barred by Treaty, U.S. General Tells Congress“, New York Times, 8.3.2017. Anfang dieses Jahres berichtete die FAZ, dass laut amerikanischen Informationen bereits vier Bataillone aufgestellt seien, vgl. Thomas Gutschker: „Russland verfügt über mehr Raketen als bislang bekannt“, Frankfurter Allgemeine Sonntagszeitung, 10.2.1019; dabei handelt es sich um ein Ausbildungsbataillon auf dem Testgelände Kapustin Jar in Südrussland, ein Bataillon in Kamyšlov, östlich von Jekaterinburg, ein weiteres im nordossetischen Mosdok sowie eines in Šuja nahe Moskau abweisende Fähigkeiten aufweisen und Reichweiten von bis zu $3.000 \mathrm{~km}$ und mehr haben. Dazu gehören neben den bekannten $K h$-55 und $K h$-55SM Marschflugkörpern die seit 2013 eingeführten Flugkörper des Typs Kh-101/102. Auch stationiert Russland ballistische Mittelstreckenraketen des Typs Iskander im Bezirk Kaliningrad, die zwar nicht unter den INF-Vertrag fallen, die aber Berlin und Warschau direkt bedrohen können. All die hier genannten Flugkörper können nuklear und konventionell bestückt werden. Hinzu kommt, dass Russland seit Jahren strategische nukleare Angriffssysteme so modifiziert, dass diese auch gegen Europa eingesetzt werden können. Hierzu gehört vor allem der noch nicht in Dienst gestellte ballistische Flugkörper RS-26 Rubezh, der mit einer Reichweite von knapp $5.800 \mathrm{~km}$ von russischem Territorium aus Ziele in Europa angreifen kann. ${ }^{4}$ Die neuesten Ankündigungen Präsident Putins, hyperschnelle und extrem manövrierfähige Raumgleiter als Kernwaffenträger einzusetzen, gibt dieser Dimension eine weitere Relevanz. Diese Gleiter können auch gegen Ziele in Europa eingesetzt werden seien sie konventionell oder nuklear bestückt.

Viele argumentieren, dass diese Marschflugkörper und Raketen vor allem einen Bezug zu China hätten. ${ }^{5}$ China würde an der Grenze zu Russland Mittelstreckenraketen aufstellen. Dieser Chinabezug wird auch von amerikanischen Autoren betont, die den Ausstieg der USA aus dem INF-Vertrag in der Hauptsache damit begründen, dass Washington die Fähigkeit behalten müsse, gegen chinesische Mittelstreckenraketen handlungsfähig zu bleiben. ${ }^{6}$ Dieses Argument hat zweifellos seine Berechtigung. Aber: wenn die Aufrüstung Russlands bei Mittelstreckenraketen und Marschflugkörpern primär Asien und andere Schauplätze im Sinn hätte, bei denen Russland als Großmacht ernst genommen werden möchte, dann bleibt die Frage unbeantwortet, warum die landgestützten Marschflugkörper des Typs $9 M 729$ gerade im europäischen Teil Russlands stationiert und zudem die Nordmeerflotte mit Jasen-U-Booten und Kalibr-Marschflugkörpern ausgerüs-

4 Vgl. den Beitrag von Stefan Hinz in diesem Heft.

5 Stratfor Worldview, "RIP INF: China, Russia, and America May Enter into a Deadly Arms Race", 2.2.2019; The National Interest Blog, https://nationalinterest.org/blog/buzz/rip-inf-china-russia-andamerica-may-enter-deadly-arms-race-43017; Ryan Browne: "What's behind Trump's decision to ditch a decades-old arms control treaty?", CNN, 22.10.2018; https://edition.cnn.com/2018/10/21/politics/trumprussia-inf-treaty-decision/index.html;

6 Keith K. C. Hui: „Analyst says US pulls out of INF pact because of China“, China Daily Mail, 6.2.2019; https://chinadailymail. com/2019/02/06/analyst-says-us-pulls-out-of-inf-pact-because-ofchina/; siehe auch Jesse Johnson: „China’s missile buildup - a threat to U.S. bases in Japan - likely a key factor in Trump plan to exit INF“, Japan Times, 22.10.2018. 


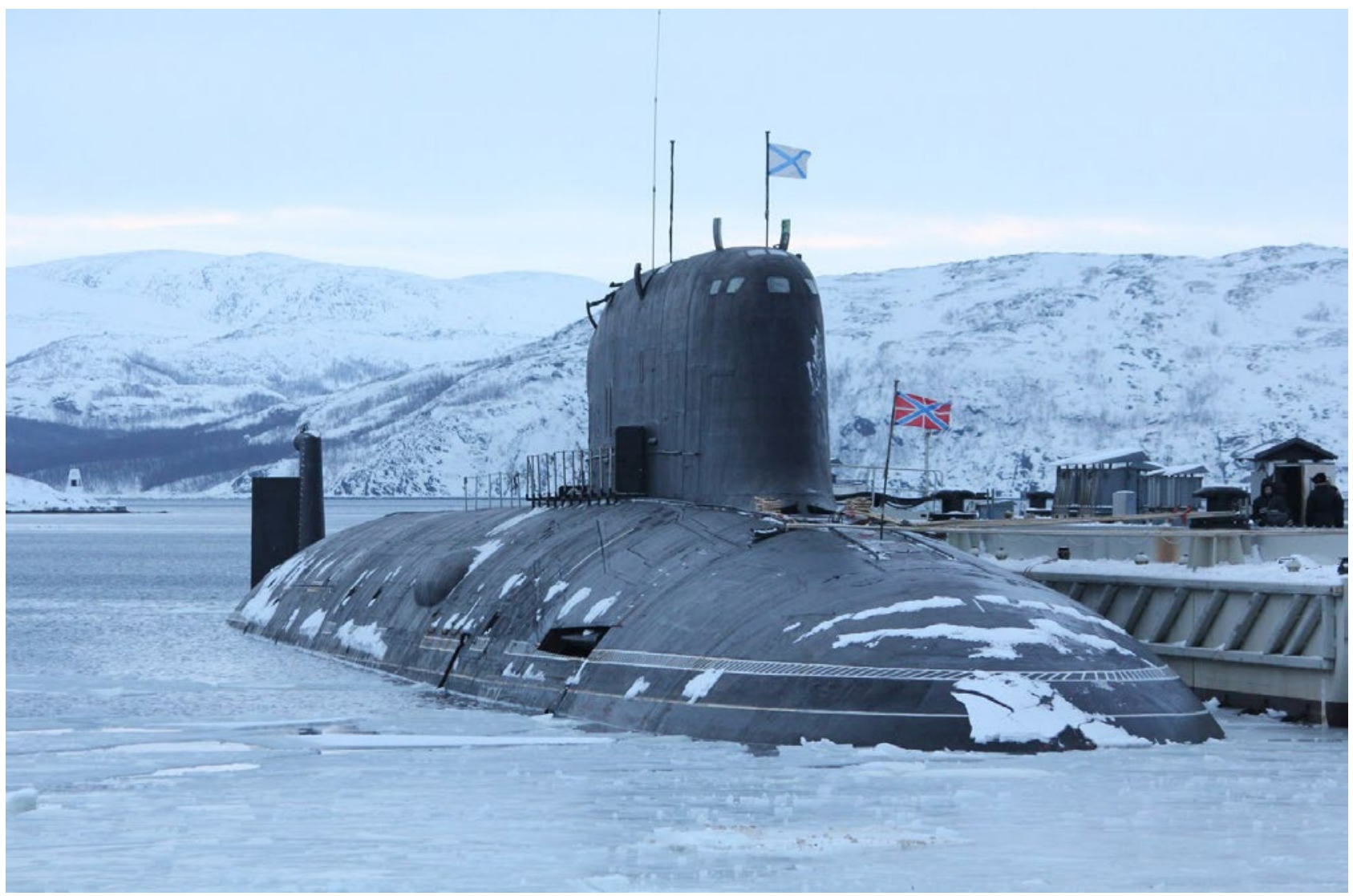

Abb. 1: Das erste U-Boot der Jasen Klasse, die Severodvinsk

tet werden und nicht etwa die Pazifikflotte. Besorgniserregend ist dabei das Ausmaß der Modernisierung und Ausweitung der russischen Lagerkapazitäten für Kernwaffen in Europa, am stärksten auf der Halbinsel Kola (dem russischen Teil Skandinaviens) und in der russischen Exklave Kaliningrad. ${ }^{7}$ All dies sind Indikatoren dafür, dass Russland in großem Umfang eine auf Europa zielende Aufrüstung mit Raketen, Marschflugkörpern und Trägersystemen betreibt, die mit relativ großer Genauigkeit Ziele in Europa aus unterschiedlichen Entfernungen treffen können - entweder mit konventionellen oder mit nuklearen Sprengköpfen.

\section{Die Motive Russlands}

Wenn Russland mit seinen begrenzten wirtschaftlichen Kapazitäten einen derart großen Aufwand betreibt, dann sollte man sich auch in Deutschland ernsthaft mit der Frage befassen, was sich die politische und militärische Führung in Moskau von diesen enormen Investitionen

7 Vgl. Nilsen 2018, Kristensen 2018. verspricht. Investitionen in Waffensysteme dieser Größenordnung werden nicht einfach so gemacht - oder weil Politiker an einer „Rüstungsschraube“ drehen wollen. Entweder stellen sie eine Reaktion auf westliche Rüstungsanstrengungen dar, oder dahinter steht ein strategisches Konzept, das auf politische und militärische Grundsatzentscheidungen zurückgeführt und auf westlicher Seite gründlich untersucht werden muss - und gegebenenfalls Gegenmaßnahmen auslösen sollte.

Die russische Aufrüstung lässt sich nicht als Antwort auf westliche Rüstungsschritte interpretieren, denn seit Anfang der 90er Jahre des 20. Jahrhunderts sind taktische und „euro-strategische“ Kernwaffen westlicher Staaten massiv reduziert worden. Die amerikanischen Pershing IIRaketen und die Marschflugkörper des Typs BGM-109G Gryphon wurden im Zuge der Implementierung des INFVertrags abgebaut und zerstört. Bis auf eine kleine Zahl von rund 200 Flugzeug-Bomben des Typs $B$-61, die in mehreren europäischen Ländern unter US-Aufsicht gelagert sind, gibt es keine amerikanischen taktischen Atomwaffen mehr in Europa. Die $B-61$ Bomben würden von Bombenflugzeugen ausgebracht, die verwundbar sind. Seit 1991 führen US-Überwasser-Kriegsschiffe keine Kern- 
waffen mehr mit sich. In diesem Zusammenhang hatten die USA auch darauf verzichtet, die sehr effektiven und zielgenauen seegestützten Tomahawk-Marschflugkörper mit nuklearen Sprengköpfen auszurüsten. Diese Festlegung ist unter dem Eindruck des russischen Bruchs des INF-Vertrags mittlerweile aufgegeben worden - ohne dass allerdings schon ein entsprechender nuklearer Flugkörper beschafft worden wäre. Auch die britischen und französischen Kernwaffenarsenale wurden drastisch reduziert. Diese befinden sich auf nukleargetriebenen U-Booten, die in offenen Gewässern operieren, oder könnten, im Falle Frankreichs, auch mit Kampfflugzeugen ins Ziel gebracht werden. Beide Szenarien sind aber realistischerweise nur vorstellbar, wenn die Sicherheit Frankreichs oder Großbritanniens von außen existentiell bedroht wäre, also durch die Drohung eines Nukleareinsatzes gegen deren Territorien. Dies ist aber sehr unwahrscheinlich, da Russland mit einem vernichtenden nuklearen Gegenschlag zu rechnen hätte. Russischen Planern ist dies sehr wohl bewusst. Dafür einen derart großen Aufwand zu treiben, wäre reine Mittelverschwendung, was den normalerweise kühl kalkulierenden russischen Militärs klar sein dürfte.

Wenn das gängige Rüstungswettlauf-Theorem die umfassende russische Aufrüstung im Bereich der eurostrategischen Raketen nicht erklären kann, dann ist es geboten, sich mit strategischen Überlegungen zu befassen, die dieser Rüstung zugrunde liegen. Hierzu sind die Merkmale der eingeführten Waffensysteme ebenso in Betracht zu ziehen wie die übrigen militärischen Aktivitäten Moskaus der vergangenen Jahre in Europa vor dem Hintergrund der politischen und strategischen Ausrichtung Russlands und seiner damit einhergehenden Militärdoktrin. Diese sieht unter anderem vor, dass konventionelle und nukleare Kräfte und Mittel einen integrierten Verbund bilden und dass auch der Einsatz von Nuklearwaffen oder die Drohung mit ihnen ein Mittel der operativen Kriegsführung darstellen. Die naheliegende Erklärung ist die, dass Russland die Fähigkeit zur Eskalationsdominanz in einem regionalen Konflikt in Europa anstrebt und sie in absehbarer Zeit erreichen könnte. Anders ausgedrückt: Russland betreibt diese enormen Investitionen, weil es sich davon entscheidende strategische Vorteile gegenüber den USA und der NATO für den Fall eines regionalen Krieges in Europa verspricht - eines Krieges, der aller Voraussicht nach nur von Russland ausgehen würde.

Das Selbstverständnis von der Einmaligkeit Russlands und der daraus erwachsene Machtanspruch, die Geschichte des Landes als einer jahrhundertelangen Geschichte der Eroberung eines Riesenreiches und seine geostrategische Lage mit einer mehr als 20.000 Kilometer langen Landgrenze begründen Moskaus Bestreben,
Russlands eigene Sicherheit absolut zu definieren, also auf Kosten der Sicherheit Anderer, vor allem von Staaten und Bündnissen in seiner Nachbarschaft. Was diesem Konzept derzeit im Wege steht, ist die NATO. Würde sie geschwächt, ihre Geschlossenheit unterminiert, ihre Entscheidungsfähigkeit in einer Krise paralysiert oder ihre Verteidigungsfähigkeit in einem Konflikt zunichtegemacht und würde die NATO daraufhin kollabieren, hätte Russland freie Bahn, und seine Kontrolle über Europa würde sich wie von selbst ausbreiten. Diesem strategischen Ziel dienen die neuen nuklearfähigen Mittelstreckenraketen 9M729 ebenso wie die Kalibr-Marschflugkörper und die luftgestützten Marschflugkörper. Die bereits bestehende vielfache konventionelle und nukleare Bedrohung erhält damit eine neue militärische und politische Qualität. Vor allem mit der 9M729 verschafft sich die russische Führung eine zusätzliche, besondere Option zur Durchsetzung ihrer Strategie mit weitreichenden Implikationen.

Mit einer Reichweite von über 2.000 km, landgestützt und mobil und daher schwer zu identifizieren und $\mathrm{zu}$ bekämpfen, kann die $9 M 729$ mit geringer Vorwarnzeit jedes Ziel im größten Teil Europas erreichen - Hauptstädte, militärische Hauptquartiere und für die Überlebens- und Verteidigungsfähigkeit unserer Länder kritische zivile und militärische Infrastruktur. Ähnliches gilt im Prinzip für die Kalibr-Marschflugkörper sowie die anderen oben genannten Mittelstreckenwaffen. Wegen der geringen Vorwarnzeit aber könnte die 9M729 die Reaktionsfähigkeit der NATO und damit die Aufmarsch- und Verteidigungsfähigkeit der Verbündeten zu einem frühen Zeitpunkt eines Konfliktes, womöglich entscheidend, schwächen und als Folge die Entscheidungsfähigkeit einzelner Alliierter oder der NATO als ganzer paralysieren. Da die $9 M 729$ zudem zwar nahezu ganz Europa bedrohen kann, nicht aber die USA, könnte hinter dieser Beschaffung die Absicht der russischen Führung stehen, die USA aus einem Konflikt in Europa herauszuhalten zu wollen, zumal wenn er eine nukleare Dimension hätte. Dies könnte die auf Europa erweiterte nukleare Abschreckung der USA unterminieren, die strategische Einheit des Bündnisgebiets aufbrechen und in zwei Zonen unterschiedlicher Sicherheit aufteilen und damit die Sicherheit Europas von der Sicherheit Nordamerikas abkoppeln - der Albtraum aller deutschen Regierungen schon im Kalten Krieg.

Der russischen Regierung ist bewusst, dass sie einen lang andauernden Krieg mit dem Westen und eine strategische Auseinandersetzung mit der NATO auf absehbare Zeit nicht gewinnen kann. Aber in der Beurteilung aller oben dargelegten Faktoren könnte Moskau zu der Auffassung gelangen, dass es eine regionale Eskalationsdominanz erlangt hat und daher das Risiko einer militärischen Aus- 
einandersetzung in Europa am Rande der NATO beherrschen und deren Handlungsfähigkeit untergraben kann. Eine solche Perzeption kann die Risikobereitschaft und Aggressivität Moskaus in einer Krise, aber bereits auch schon in Friedenszeiten deutlich erhöhen. Der Zwischenfall im Asovschen Meer könnte dafür ein Beispiel sein. Sollte es in Europa zu einem regionalen Krieg kommen, würde Moskau alles daransetzen, ihn zu seinen Gunsten $\mathrm{zu}$ entscheiden und mit Hilfe dieser Mittelstreckenwaffen zu beenden - entweder konventionell oder auch nuklear.

Das hört sich für deutsche Ohren erst einmal befremdlich an, aber vieles spricht dafür, dass die russische Führung davon ausgeht, dass es in den kommenden Jahren zu einer kriegerischen Auseinandersetzung in Europa kommen kann. Dies steht auch im Einklang mit wissenschaftlichen Analysen, die sich mit dem spezifisch russischen Ansatz von Kriegsführung und von Anwendung von Gewalt generell befassen. Die Verteidigungspolitik der westlichen Allianz hat sich in den vergangenen 70 Jahren auf Abschreckung durch nukleare Vergeltungsandrohung und/oder durch Versagen von militärischen Optionen mit konventionellen Streitkräften im Rahmen der Kollektiven Verteidigung beschränkt. In den zurückliegenden drei Jahrzehnten war sie zudem vor allem durch Interventionen zur Stabilisierung von Krisenherden und Konflikten außerhalb der Bündnisgrenzen im Sinne kollektiver Sicherheit (meist gemischt militärisch-zivil) gekennzeichnet.

Demgegenüber war der sowjetische und ist heute auch der russische Ansatz zum Einsatz militärischer Gewalt grundlegend anders. Dieser ist durch das oben beschriebene russische Selbstverständnis geprägt, das faktisch die Unterwerfung der Sicherheitsinteressen seiner Nachbarstaaten unter den russischen Machtanspruch verlangt. Hinzu kommt ein militärstrategisches Denken, das Kriege antizipiert, die gewonnen werden müssen unter Ausnutzen der Schwächen der anderen Seite bei Vermeidung der Ausnutzung der eigenen Schwächen durch den Gegner. Russisches Denken geht (abgesehen von der nuklearstrategischen Konfrontation mit den USA) nicht von dem Primat der Vermeidung und raschen Beendigung von Kriegen und der Schadenslimitierung aus, sondern davon, Kriege zu gewinnen - und auch nur jene Kriege zu initiieren, bei denen man sicher ist, sie auch gewinnen zu können. Dabei wird das gesamte Spektrum an militärischen und nicht-militärischen Instrumenten in Betracht gezogen. Dieses Spektrum umfasst Desinformationskampagnen, Destabilisierungsoperationen und CyberAngriffe schon in Friedenszeiten sowie hybride Angriffe, die unterhalb der Schwelle von offener Anwendung von militärischer Gewalt von außen bleiben, und schließlich klassische konventionelle Operationen zur Besetzung und Verteidigung von Territorien bis hin zum Einsatz nuklearer Waffen. ${ }^{8}$

Kriege werden in ihren Konsequenzen und in Bezug auf das Eintreten möglicher Ereignisse (auch das Einschreiten dritter Parteien) voll durchdacht. Wie in einem Schachspiel stellt sich die russische Führung dabei auf unterschiedliche Szenarien und Strategien eines Gegners ein. Einen hohen Stellenwert nehmen dabei regionale Kriege an der Peripherie Russlands ein. Zentral ist die Frage, wie diese unter Nutzung von unterschiedlichen Mitteln zu Gunsten Russlands erfolgreich beendet werden können. Dabei wird in der russischen Militärdoktrin auch Kernwaffen eine zentrale Rolle bei der Eskalationskontrolle zugewiesen, wenngleich das übergeordnete Ziel bleibt, den umfassenden strategischen Kernwaffenkrieg $\mathrm{zu}$ vermeiden. ${ }^{9}$ In diesem Zusammenhang werden auch nicht-nukleare Präzisionswaffen in das militärische Kalkül einbezogen. Bei ihnen handelt es sich hauptsächlich um Marschflugkörper und ballistische Raketen kurzer und mittlerer Reichweite, die entweder land- oder seegestützt sind oder von Flugzeugen aus gestartet werden. Solche weitreichenden konventionellen Waffen, die auch mit nuklearen Gefechtsköpfen bestückt werden können, sollen in einem regionalen Krieg den Gegner zum Aufgeben zwingen. ${ }^{10}$

\section{Regionale Kriege in Europa - welche Schauplätze?}

Aber wo könnten derartige Kriege stattfinden? Und wären dort auch aus russischer Sicht legitime defensive Interessen betroffen? Oder würde dort die gewaltsame Umsetzung der revisionistischen Politik Putins beginnen, der wiederholt die Auflösung der Sowjetunion als größte Katastrophe des 20. Jahrhundert bezeichnet hat? Defensive Interessen Russlands wären nur dann als gegeben anzusehen, sollte es größere Truppenkonzentrationen der NATO an Russlands Grenzen geben, die zu großräumigen Angriffsoperationen fähig wären und deren Aktivitäten als Vorbereitung einer Invasion Russlands gewertet werden könnten. Diese gibt es aber weder im Baltikum noch in Skandinavien noch in Polen, in der Ukraine oder im Kaukasus.

8 Covington 2016, Adamsky 2015, Johnson 2018, Johnson 2019, Peters/Anderson/Menke 2018, Kroenig 2018, Giles 2016, Palmer 2018, Dick 2019.

9 Johnson 2018, 16 und 20, Warden 2018, 13, Kroenig 2018.

10 Warden 2018, 13f, Johnson 2018, 26. 
Die möglichen Schauplätze eines regionalen Krieges lägen dort, wo Russland glaubte, seinen Einfluss wieder herstellen zu müssen oder wo es die Chancen sieht, eine radikale Änderung des strategischen Status quo durch eine nachhaltige Schwächung der NATO zu bewirken, und zwar mit begrenztem oder beherrschbarem militärischem und politischem Risiko. Eine solche Situation könnte eintreten, wenn die USA mehr und mehr Teile ihrer Kräfte nach Ostasien verlegten, wo diese dann im Südchinesischen Meer und im Pazifik gebunden wären. Wenn sich gleichzeitig die wirtschaftliche Lage in Russland weiter verschlechtert und die Zustimmung der russischen Bevölkerung zum Regime schwindet, könnte der russische Präsident Vladimir Putin die Flucht nach vorne antreten, indem er mit einer entscheidenden militärischen Operation und mit der damit verbundenen Mobilisierung nationalistischer Emotionen in der russischen Bevölkerung seine Herrschaft wieder zu festigen versuchte. Das ist derzeit zwar nur ein theoretisches Szenario, aber es passt zu den zu beobachtenden Rüstungsentwicklungen auf Seiten Russlands.

Derzeit kommen zwei regionale Schauplätze für solche Vorstöße in Betracht: entweder (1) die Ausdehnung der militärischen Operationen in der Ukraine mit dem Ziel, deren Lebensfähigkeit als unabhängiger Staat noch stärker zu bedrohen als bisher oder deren unabhängige Staatlichkeit zu zerstören; oder (2) ein begrenzter Angriff gegen einen oder mehrere baltische Staaten und Teile Polens mit dem Ziel, die Glaubwürdigkeit der NATO und der kollektiven Verteidigungsgarantie zu unterminieren und damit ihre Rolle und Funktion zu diskreditieren. In beiden Fällen würde Russland vermutlich versuchen, mit schnellen Vorstößen militärische Fakten, ein fait accompli, zu schaffen, bevor der Westen, die USA oder die NATO als ganze eingreifen könnten. In beiden Fällen würden russische Mittelstreckenwaffen eine wichtige Rolle spielen.

\subsection{Der Schauplatz Ukraine}

Schon während der Sitzung des NATO-Russland-Rats auf Ebene der Staats- und Regierungschefs im Rahmen des NATO-Gipfeltreffens 2008 in Bukarest sprach der russische Präsident Putin - voller Zorn über die Zusage der Allianz an die Adresse der Ukraine und Georgiens, dass beide eines Tages NATO-Mitglied würden - in Anwesenheit der Staatschefs der Allianz der Ukraine eine eigene Staatlichkeit ab und kündigte mit drohendem Unterton an, dass Russland überall da seiner Verantwortung nachkommen würde, wo russische Bürger lebten und deren
Sicherheit womöglich bedroht sei. ${ }^{11}$ Es herrschte betretene Stille im Raum. Kurze Zeit danach marschierte Russland in Georgien ein. Die Folgen sind bekannt.

Heute steht Russland mit Streitkräften im Donbass und bindet dort beträchtliche Landstreitkräfte der Ukraine. Es hat die Halbinsel Krim besetzt und unrechtmäßig annektiert und zu einer großen militärischen Festung ausgebaut. Die dortigen vielfachen Luftverteidigungssysteme, die Marschflugkörper, die konventionellen oder nuklearen (ballistischen) Kurz- und Mittelstreckenraketen gegen Boden-, Luft-, und Seeziele, die weitreichende Artillerie und die elektronischen Kriegsführungssysteme decken als sogenannte Anti Access/Area Denial (A2/AD) Capabilities große Teile des Schwarzen Meeres, den nördlichsten Teil der Türkei und den östlichsten Teil Rumäniens ab. Russland kontrolliert die Straße von Kerč und damit den Zugang zum Asovschen Meer. Es hat noch immer Streitkräfte im von Moldawien abtrünnigen Transnistrien im Südwesten der Ukraine stationiert. Außerdem ist Russland in der Lage, sehr rasch größere Verbände an Landstreitkräften an der Grenze zur Ukraine zusammenzuziehen. So sähe die militärische Ausgangslage für eine weitere militärische Operation gegen die Ukraine aus.

Als ein Denkmodell für eine Fortsetzung der russischen Intervention in der Ukraine kann man sich das folgende generische, aber realitätsnahe Szenario vorstellen: Künstlich herbeigeführte Zwischenfälle und Provokationen aus dem Instrumentenkasten der hybriden Kriegsführung, wie man sie seit den Tagen der Besetzung der Krim kennt, groß angelegte, systematische Desinformationskampagnen, geschürte Unruhen in von Russen bewohn-

11 Über die Rede ist in den Medien berichtet worden, wobei der Schwerpunkt auf der Warnung Putins vor einer weiteren Erweiterung der NATO lag. Die Infragestellung der Eigenständigkeit der Ukraine wurde dabei kaum erwähnt; vgl. „Putin warns Nato over expansion“, The Guardian, 4.4.2008, sowie Steven Erlanger: „Putin, at NATO Meeting, Curbs Combative Rhetoric“, New York Times, 5.4.2009. Die Rede ist im Volltext nirgendwo mehr zu finden, weder auf der Webseite der NATO noch auf der Webseite des russischen Präsidenten. Lediglich auf der Webseite der ukrainischen Nachrichtenagentur UNIAN finden sich jene Ausschnitte der Rede Putins im englisch übersetzten Wortlaut, in denen er die Lage in der Ukraine als extrem kompliziert darstellt und ein Bild von der Ukraine malt, welches ihr weitgehend jegliche Eigenständigkeit abspricht. Putin wies in dieser Rede darauf hin, dass in der Ukraine 17 Millionen Russen leben würden; https://www. unian.info/world/111033-text-of-putins-speech-at-nato-summitbucharest-april-2-2008.html. Teilweise hat Putin auch abweichend vom Redemanuskript gesprochen. Das betrifft insbesondere die oben erwähnten Drohungen. Der Bericht über die mündlichen Einlassungen Putins und dessen Wirkung auf die anwesenden Staats- und Regierungschefs der NATO in der Sitzung des NATO-Russland-Rats in Bukarest stammt von einem damaligen hochrangigen Mitglied des Internationalen Stabs der NATO, der an der Sitzung teilnahm. 
ten Landesteilen durch eingesickerte Untergrundkämpfer und ,Aufständische‘, eine Verschärfung des Krieges im Osten, herbeigeführte Zwischenfälle im Asovschen Meer und behauptete Angriffsvorbereitungen der Ukraine gegen Russland würden von der russischen Führung als Vorwand genommen, um die militärischen Operationen gegen die Ukraine großflächig auszuweiten. Beispielsweise könnten russische Landstreitkräfte (unter Sperrung der Straße von Kerč und des See- und Luftraums über dem Asovschen Meer und bei Bindung starker ukrainischer Streitkräfte im Osten und entlang der Grenze zu Russland) versuchen, von Osten aus schnell über Mariupol hinaus vorzustoßen, die Landverbindung zur Krim zu schließen und den Raum südlich des Dnjepr zu besetzen. Sie könnten, koordiniert mit den Kräften auf der Krim und gegebenenfalls in Transnistrien weiter in Richtung Odessa vorstoßen, dem größten und wichtigsten Hafen der Ukraine, ihn auch von See aus blockieren und das Land damit von einer seiner entscheidenden Lebensadern abtrennen. Die Regierung in Kiev könnte vor die Wahl gestellt werden: entweder die Fortsetzung des Krieges und damit die Zerstörung und Besetzung weiter Teile des Landes oder die Assoziierung mit Russland. ${ }^{12}$

Die gesamte Operation würde unterstützt und abgesichert einerseits durch zielgenaue konventionelle Raketen aus der Tiefe des russischen Raums, die wichtige militärische Ziele und zivile Infrastruktur in der Ukraine bekämpfen würden, die für die Operationen der ukrainischen Streitkräfte von wesentlicher Bedeutung wären. Außerdem wäre die russische Führung vermutlich bestrebt, sofort mit Beginn der Operationen die militärische Kontrolle über das Schwarze Meer und dessen Luftraum zu erreichen - gegen angenommene mögliche westliche Interventionsversuche.

Darüber hinaus könnte die russische Führung den Zugang zum Schwarzen Meer sperren. Sie könnte Polen und Rumänien oder sogar Zentraleuropa in die Zielliste für nuklearbestückte Mittelstreckenraketen aufnehmen und die betroffenen Nationen, aber auch die USA und die NATO, gezielt darüber in Kenntnis setzen. Putin wäre auf allen Kanälen mit der Warnung zu vernehmen, dass Russland eine Nuklearmacht sei und sich nicht scheuen würde, seine nationalen Interessen mit allen Mitteln zu verteidi-

12 Würde sich die ukrainische Regierung allerdings weigern, sich einer russischen Erpressung zu beugen, stünde Moskau möglicherweise ein längerer, verlustreicher Zermürbungskrieg bevor, der von Kiev womöglich mit Guerilla-Taktiken geführt würde, auch zu Anschlägen in Russland führen würde und schließlich die USA auf den Plan rufen könnte. Deshalb würde Russland alles daransetzen, sehr schnell vollendete Tatsachen zu schaffen und die Ukraine militärisch zu strangulieren. gen. Die Botschaft wäre klar: Jede Einmischung von westlicher Seite mit militärischen Mitteln könnte zu nuklearer Eskalation mit dramatischen Folgen führen.

Wäre Russland damit erfolgreich, würde sich die gesamte strategische Lage in Südosteuropa und im gesamten Schwarzmeerraum, einschließlich der Türkei, und die dortige Kräftekonstellation grundlegend verändern. Rumänien sähe sich einer direkten militärischen Bedrohung gegenüber, und die gesamte NATO-Verteidigungsplanung müsste angepasst werden. Der Aufbau einer starken permanenten militärischen Präsenz des Bündnisses entlang seiner gesamten Ostflanke wäre das Gebot der Stunde. Moskau hätte allerdings seine Pufferzone gegenüber der Allianz eingebüßt.

\subsection{Der Schauplatz Baltikum}

Ein analoges Vorgehen der russischen Führung im baltischen Raum wäre selbst im Falle einer strategischen ,Ablenkung' der USA in Ostasien ungleich risikoreicher, aber nicht auszuschließen. Die baltischen Staaten, Polen, Deutschland und Dänemark sind NATO-Mitglieder. Für sie gälte die Beistandsgarantie des Washingtoner Vertrags. Schweden und Finnland sind NATO-Partner und durch koordinierte Übungen und Einsatzplanung eng mit der NATO verbunden. ${ }^{13}$ Die USA haben starke Land-, Luft- und Seestreitkräfte in Europa stationiert, vor allem in Deutschland und Polen. Sie sind auch zu Übungen mit Land-, Luftund Seestreitkräften regelmäßig in den baltischen Staaten und im Ostseeraum insgesamt präsent.

Die Streitkräfte Estlands, Lettlands, Litauens und Polens sind auf die Verteidigung ihrer Länder vorbereitet. Die vier multinationalen Battlegroups der NATO dort signalisieren der Moskauer Führung, dass sie selbst im Falle eines begrenzten Einfalls in eines dieser Länder unmittelbar in einen militärischen Konflikt mit der gesamten Allianz geriete, einschließlich der drei nuklearen Mächte der NATO: USA, Frankreich und Großbritannien. NATOKampfflugzeuge sichern ständig den Luftraum über den baltischen Staaten. Außerdem hat die NATO seit der hybriden Aggression Russlands gegen die Ukraine im Jahre 2014 ihre Abschreckungs- und Verteidigungsfähigkeit systematisch verstärkt und ihre Verteidigungsausgaben deutlich erhöht: Seit 2014 haben die europäischen Verbündeten und Kanada rund 88,5 Milliarden US-Dollar mehr für Verteidigung ausgegeben, bis Ende 2020 werden es weit mehr als 100 Milliarden US-Dollar sein. Allein die USA haben im Rahmen ihrer European Defence Initiative

13 Vgl. Hedlund 2017. 
in diesem Jahr 6,5 Milliarden US-Dollar für mehr Truppen, Übungen, vorausstationierte Ausrüstung und für Infrastruktur aufgewandt. ${ }^{14}$

Gleichwohl genießt Russland einige wichtige, geographisch bedingte, militärische Vorteile. Die baltischen Staaten und Polen haben eine gemeinsame Grenze mit Russland. Die russischen Streitkräfte sind so disloziert, dass starke Verbände rasch über relativ kurze Entfernungen überallhin an die russische Westgrenze verlegt und dort konzentriert werden können. NATO-Truppen, die Alliierte am Rande des Bündnisgebiets im Norden, Nordosten, Osten und Südosten verstärken sollten, müssten dagegen mehrere Landesgrenzen und größere Entfernungen überwinden, um in den Einsatzraum zu gelangen, mit allen damit verbundenen militärischen Risiken. Russland genießt also zunächst einen Raum-Zeit-Kräfte-Vorteil. Außerdem könnte Russland den Aufmarsch der NATO zur raschen Verstärkung im Osten unter Kriegsbedingungen durch seine A2/AD-Fähigkeiten verzögern und schwächen. Dazu hat es die entsprechenden Fähigkeiten im Oblast Kaliningrad, der wie ein Keil ins NATO-Territorium hineinragt, und auch im Rahmen der Baltischen Flotte. Das Suwalki Gap zwischen Kaliningrad und Weißrussland wirkt wie ein Flaschenhals für den Zugang von NATO-Streitkräften zu den baltischen Staaten und könnte womöglich das erste Ziel eines russischen Angriffs sein, um diese vom restlichen NATO-Territorium abzuschneiden.

Käme die russische Führung zu dem Schluss, dass die gesamte strategische Lage einen militärischen Angriff mit begrenztem Ziel, aber weitreichenden politischen und strategischen Wirkungen erlaubt oder sogar begünstigt und das damit verbundene Risiko beherrschbar wäre, könnte sie sich zu einem schnellen Vorstoß mit konventionellen Streitkräften in die baltischen Staaten und einen Teil Polens entschließen. Ziel wäre es, die baltischen Staaten von Kaliningrad aus von der restlichen NATO abzutrennen. Mit weitreichenden konventionellen Mittelstreckenwaffen könnte Moskau versuchen, wichtige Ziele auszuschalten, die für den Aufmarsch von NATO-Streitkräften in Europa und über den Atlantik hinweg zur Verstärkung der angegriffenen Verbündeten von wesentlicher Bedeutung wären. Drohungen mit dem Einsatz von nuklearen Mittelstreckenwaffen würden dazu dienen, europäische Regierungen zu entmutigen, ihre Streitkräfte einzusetzen. Diese Drohungen könnten desto glaubhafter sein, je größer die Unsicherheiten unter den europäischen Staaten darüber wären, wie glaubhaft die amerikanische nukleare Sicherheitsgarantie für ihre Verbündeten noch ist. Hätten russi-

14 Meyer zum Felde 2018. sche Streitkräfte die baltischen Staaten oder Teile davon besetzt, bevor NATO-Verstärkungskräfte entscheidend eingreifen könnten, könnte Moskau innehalten und die NATO vor die Wahl stellen, entweder einen großen Krieg zu riskieren mit unberechenbarer Eskalationsmöglichkeit oder klein beizugeben und zuzustimmen, dass die baltischen Staaten - im besten Fall - die NATO verließen und ,neutral' würden. Die politischen Folgen für die NATO und die Sicherheit Europas wie auch für das transatlantische Bündnis dürften dramatisch sein. Es könnte das Ende der Allianz einläuten.

\section{Welche Gegenmaßnahmen sind notwendig?}

Bei der Beschreibung der beiden generischen Szenarien sollte klargeworden sein, dass man die Aufstellung der neuen russischen Mittelstreckenwaffen nicht isoliert betrachten darf, sondern im Zusammenhang der gesamten russischen Strategie in Frieden, Krise und Krieg analysieren muss. Diese Waffen haben einen militärischen Zweck im Verbund mit russischer konventioneller Kriegsführung, sie haben als ein Instrument der russischen Konfliktstrategie gegenüber dem Westen aber auch eine strategische und politische Wirkung, die sich in einer Krise entfaltet und daher bereits im Frieden spürbar werden kann - durch Einschüchterung mit dem Verweis auf die Existenz der Waffen und deren Parameter und Kapazitäten, durch Nötigung oder Erpressung mit der Drohung ihres Einsatzes in Krise und Krieg. Daraus folgt, dass seitens der NATO Gegenmaßnahmen notwendig werden, mit denen die strategische und politische Wirkung dieser Waffen neutralisiert werden kann. Im Wesentlichen geht es darum, die Vorstellung Moskaus zu konterkarieren, Russland besäße unter den genannten Szenarien die Fähigkeit zur militärischen und politischen Eskalationsdominanz. Im Einzelnen geht es darum, das Risikokalkül Moskaus gezielt und entschlossen zu beeinflussen. Bei der Analyse von Gegenmaßnahmen der NATO ist es geboten, den Gesamtzusammenhang der Abschreckungs- und Verteidigungsstrategie der NATO mit ihren konventionellen und nuklearen Komponenten im Blick zu behalten. Da sich die Bedrohung durch die neuen Mittelstreckenwaffen (sowohl konventionell als auch nuklear) in erster Linie im Kontext konventionell oder hybrid geführter Eroberungsoperationen der russischen Streitkräfte im Rahmen regionaler Kriegsszenarien ergibt, sollten Gegenmaßnahmen in erster Linie darauf abzielen, Russland die Fähigkeit zu 
versagen, derartige Operationen erfolgreich zu führen. ${ }^{15}$ Was die konventionelle Komponente der NATO-Strategie betrifft, gilt es alles zu unternehmen, um die Fähigkeit zur raschen, wirkungsvollen Verstärkung von Alliierten $\mathrm{zu}$ gewährleisten, wenn diese bedroht würden. Das gilt vor allem für diejenigen, die eine gemeinsame Grenze mit Russland haben und sich einer möglichen direkten militärischen Bedrohung ausgesetzt sehen, insbesondere das Baltikum. Mit Blick auf die Ukraine und die Schwarzmeerregion ist die Herausforderung anders gelagert. Während die Ukraine kein Bündnismitglied ist und daher nicht die kollektive Verteidigungsgarantie der NATO genießt, ${ }^{16}$ könnten die EU, Kanada und die USA eine politische Drohkulisse aufbauen, die vor allem Sanktionen beinhalten sollte, die Russland massiv treffen würden.

Die Allianz hat auf ihrem Gipfel in Brüssel im Jahr 2018 eine Fülle von Entscheidungen getroffen, die der weiteren Verbesserung der Abschreckung und, wenn nötig, Verteidigung gegen konventionelle oder hybride Bedrohungen dienen und die nunmehr umgesetzt werden. ${ }^{17}$ Von ihnen profitiert vor allem die Sicherheit im Baltikum und auch in der Schwarzmeerregion, sie dienen aber auch der Sicherheit der gesamten NATO. Umgekehrt hängt die Sicherheit im Baltikum und in der Schwarzmeerregion entscheidend von der Kohärenz und Glaubwürdigkeit der Sicherheitsvorsorge der Allianz insgesamt ab, so dass es in ihrem gesamten Verantwortungsbereich - von Nord-Norwegen, dem Nordatlantik und Atlantik, über den Ostsee- und Schwarzmeerraum, bis hin zur Mittelmeerregion, Nordafrika und dem Nahen Osten - keine wesentlichen Lücken gibt, die Russland in einer Krise ausnutzen könnte, um die NATO zu erschüttern oder zu erpressen.

Die Brüsseler Entscheidungen gehen davon aus, dass sich die NATO in mehreren Regionen mit Widersachern, Risiken und Bedrohungen unterschiedlicher Art konfrontiert sieht, die überall dort unverhofft, quasi über Nacht, und gleichzeitig auftreten können. Dies erfordert ein Höchstmaß an Reaktionsfähigkeit, Flexibilität und Einsatzbereitschaft, um mit den richtigen Kräften zur rechten Zeit am richtigen Ort sein zu können. Einige der wichtigsten Maßnahmen, um diesen Imperativ umzusetzen, sind im Folgenden aufgeführt:

15 Siehe auch Elbridge Colby/Walter Slocombe: „Eine Landnahme durch Moskau verhindern“, Frankfurter Allgemeine Zeitung (FAZ) 3.4.2019, S. 8 („Fremde Federn“).

16 Theoretisch könnte die Ukraine die NATO um Hilfe ersuchen und die NATO darauf positiv antworten; diese Option dürfte aber derzeit politisch unwahrscheinlich sein.

17 Brauß 2018.
1. Die Reaktionsfähigkeit der Nato wird weiter erhöht, indem Aufklärung und Alarmierung verstärkt und die Entscheidungsverfahren weiter beschleunigt werden.

2. Die Kommandostruktur der NATO, also das Netz ihrer militärischen Hauptquartiere, wird wieder befähigt, Verteidigungsoperationen mit Großverbänden, nun auch unter Cyber-Bedrohung, zu führen. Zwei neue Hauptquartiere, das Joint Force Command in Norfolk/ Virginia und das Joint Support and Enabling Command in Ulm werden für das Management von Truppenbewegungen über den Atlantik und quer durch Europa zuständig - amerikanisch-deutsche Kooperation im Dienst der NATO.

3. Cyber-Abwehr und die Fähigkeit, Cyber-Operationen zu führen, werden weiter verstärkt.

4. Die Einsatzbereitschaft der alliierten Streitkräfte wird weiter verbessert. Bereits im Jahr 2015 wurde der Umfang der NATO Response Force auf rund 40.000 Mann verdreifacht und ihre sehr schnelle Speerspitze aufgestellt, die Very High Readiness Joint Task Force von rund 5.000 Mann. Nun wird mit der NATO Readiness Initiative 4-30 bis 2020 die Einsatzbereitschaft von weiteren 30 Bataillonen, 30 Kampfflugzeugstaffeln und 30 Kriegsschiffen so erhöht, dass sie in maximal 30 Tagen im Einsatzraum sein können. Sie werden zu hoch einsatzbereiten Brigaden, Geschwadern und maritimen Einsatzgruppen weiterentwickelt.

5. Die Bedingungen für die rasche Verlegung alliierter Truppen innerhalb Europas über Ländergrenzen hinweg werden durchgreifend verbessert - für Übungen im Frieden und zum raschen Aufmarsch von NATO-Truppen, vor allem auch von US- und kanadischen Streitkräften, in einer sich zuspitzenden Krise. NATO und EU arbeiten dazu eng zusammen. Die Europäische Kommission stellt viele Milliarden Euro für die Verbesserung von Infrastruktur bereit und leistet damit auch einen wichtigen Beitrag zur transatlantischen Lastenteilung.

Mit Blick auf die baltischen Staaten und Polen muss vor allem der Raum-Kräfte-Zeit-Vorteil Russlands ausgeglichen werden. ${ }^{18}$ Die multinationalen Battlegroups in den baltischen Staaten und Polen sollten verstärkt werden mit Kampfunterstützungskräften wie Artillerie und Flugabwehr, und auch in Estland, Lettland und Litauen sollten sie eine permanent präsente amerikanische Kampfkomponente erhalten, die den Abschreckungswert der Battlegroups noch einmal erhöhen würde. NATO-Luftstreitkräfte sind die Verstärkungskräfte der ersten Stunde. Sie müssen

18 Vgl. Vershbow/Breedlove 2019, Kramer/Binnendijk 2018 
verzugslos eingesetzt werden können und dies vermehrt und demonstrativ üben. Für die rasche Verlegung von Verstärkungskräften käme der Luftverteidigung und Raketenabwehr der NATO und der einzelnen Verbündeten eine zentrale Rolle zu. Sie muss im Lichte der neuen Bedrohung aus Russland angepasst und neu ausrichtet werden. Zudem sollte die Beschaffung von Flug- und Raketenabwehrsystemen kurzer und mittlerer Reichweite für alle europäischen Verbündeten hohe Priorität erhalten. Diese waren in den Jahren vor 2014 stark reduziert worden, als sich die NATO auf Kriseneinsätze praktisch ohne Luftbedrohung konzentrierte. Die sichtbare maritime Präsenz der NATO auf der Ostsee, einschließlich mit Kriegsschiffen der USA, sollte deutlich erhöht werden, um zu demonstrieren, dass die NATO nicht gewillt ist, in einer Krise und im Konfliktfall der russischen Baltischen Flotte die Kontrolle über die Ostsee zu überlassen. Dies ist auch wichtig, um im Konfliktfall die A2/AD-Glocke über dem Oblast Kaliningrad mit koordinierter Wirkung aus der Luft, von See und von Land aus ausschalten zu können. Gemeinsame Übungen und koordinierte Planung mit Schweden und Finnland sollten weiter intensiviert werden.

Alle Entscheidungen und Maßnahmen, welche die NATO jetzt zur Stärkung ihres konventionellen Abschreckungs- und Verteidigungsdispositivs ins Werk setzt, dienen auch der Sicherheit der Verbündeten in der Schwarzmeer-Region: Rumänien, Bulgarien und der Türkei. Es kommt darauf an, die Kohärenz von Abschreckung und Verteidigungsvorsorge entlang der gesamten Ostflanke der NATO zu gewährleisten. Die Aufklärungstätigkeit der NATO im und über dem Schwarzen Meer sollte intensiviert und perpetuiert und die militärische Übungspräsenz der NATO, vor allem der USA, zu Lande, in der Luft und auf See, erhöht werden. Allerdings wird dies durch den Vertrag von Montreux von 1936 erschwert, der die Größe und Dauer des Aufenthaltes von Kriegsschiffen im Schwarzen Meer von Staaten begrenzt, die nicht Anrainer sind - und die Türkei hat bisher auf ihrer alleinigen Zuständigkeit für die Implementierung des Vertrags gepocht. In jedem Fall sollten Übungen der alliierten Luftstreitkräfte und die Verstärkung der südöstlichen NATO-Verbündeten mit Landstreitkräften regelmäßig und demonstrativ geübt werden.

Die Aufstellung der russischen nuklearfähigen Mittelstreckenwaffen machen die rasche Umsetzung aller oben beschriebenen Maßnahmen zur Stärkung des konventionellen Dispositivs der NATO und die Verabschiedung einer kohärenten Militärstrategie noch dringlicher. Natürlich dürfen auch, was die nukleare Komponente der Abschreckungsstrategie der NATO betrifft, der Bruch des INF-Vertrags und die Aufstellung der 9M729 durch Russ- land nicht unbeantwortet bleiben. Die Allianz ist dabei, Optionen zu prüfen, die die strategische Wirkung dieser Waffen glaubwürdig konterkarieren können. ${ }^{19}$ Dabei kommt es darauf an, die Geschlossenheit des Bündnisses zu wahren und die Glaubwürdigkeit der Abschreckung der NATO als ganzer zu erhalten ebenso wie die der erweiterten nuklearen Abschreckung der USA. Es kommen aus europäischer Sicht daher nur Lösungen in Betracht, die die strategische Einheit des Bündnisgebiets über den Atlantik hinweg wahren und die Anbindung des Abschreckungs- und Verteidigungsdispositivs der NATO in Europa an das nuklearstrategische Potential der USA gewährleisten. Die Glaubwürdigkeit von Abschreckung erfordert, dass die Allianz jederzeit über ein ganzes Spektrum an Optionen verfügt, aus dem diejenigen aktiviert würden, welche die russische Führung in einer Krise am wahrscheinlichsten von einem militärischen Angriff abhalten und damit auch die Drohung entkräften könnten.

Die USA planen, der auf Europa konzentrierten russischen Nukleardrohung kurzfristig mit seegestützten Raketen mit nuklearen Gefechtsköpfen von begrenzter Sprengkraft entgegenzuwirken. Diese Waffen werden zurzeit produziert. ${ }^{20}$ Langfristig ist die Beschaffung von seegestützten Marschflugkörpern (SLCM) geplant. ${ }^{21}$ Darüber hinaus werden offenbar die Entwicklung und Aufstellung landgestützter, zielgenauer Mittelstreckenwaffen mit hochwirksamen konventionellen Gefechtsköpfen in Betracht gezogen, die russische Führungseinrichtungen bedrohen und die militärische Handlungsfähigkeit Russlands lähmen könnten. ${ }^{22}$ Generell muss gelten: Alle zielführenden Optionen müssen ergebnisoffen analysiert und die optimale dann ausgewählt werden, keine darf aus kurzsichtigen oder opportunistischen Gründen vorschnell zur Seite gelegt werden. Vorfestlegungen einzelner Regierungen auf eine bestimmte Option oder der Ausschluss einer anderen schaden der Geschlossenheit des Bündnisses und können die Sicherheit Europas unterminieren statt sie zu vermehren. Auch die Entwicklung landgestützter nuklearer Mittelstreckenwaffen sollte man nicht von vornherein ausschließen. Sie können Moskau möglicherweise am ehesten an den Verhandlungstisch bringen, weil von ihnen die Botschaft ausginge, dass russisches Territorium

19 Brauß/Mölling 2019.

20 "W76-2 supports national security initiative requested by the President in the 2018 Nuclear Posture Review", Pressemeldung der National Nuclear Security Administration vom 25.2.2019; https:// www.energy.gov/nnsa/articles/nnsa-completes-first-productionunit-modified-warhead.

21 Department of Defense 2018, $54 \mathrm{f}$.

22 Paul Sonne: "U.S. military to test missiles banned under faltering nuclear pact with Russia,” Washington Post 13.3.2019. 
kein Sanktuarium bleibt, wenn Russland Europa nuklear bedroht. Dagegen scheinen in Deutschland viele Beobachter und Politiker eine größere Furcht vor möglichen Gegenmaßnahmen der NATO zu haben, die noch gar nicht entschieden geschweige denn realisiert sind, als vor den russischen Mittelstreckenraketen, die jetzt schon Berlin bedrohen können.

Schließlich muss der russischen Führung immer wieder durch angemessene strategische Kommunikation klar gemacht werden, dass die NATO ein nukleares Bündnis ist und bleibt und dass jeglicher Einsatz von Nuklearwaffen gegen das Bündnis die Natur eines Konflikts fundamental verändern würde, der dann nicht mehr beherrschbar wäre und Russland selbst einen unakzeptablen hohen Schaden zufügen könnte, der in keinem Verhältnis zu dem angestrebten militärischen und politischen Gewinn stünde. ${ }^{23}$

\section{Fazit}

Die Motive hinter den massiven russischen Rüstungsanstrengungen im Bereich präziser Mittelstreckenwaffen in Europa (die sowohl konventionell als auch nuklear bestückt werden können) erschließen sich nicht durch das in der deutschen politischen Debatte immer wieder bemühte Aktions-Reaktions-Theorem. Sie sind nicht die Folge westlicher Rüstungsanstrengungen oder politischer Missverständnisse, sondern sie sind Konsequenz eines strategischen Konzepts der russischen politischen und militärischen Führung, welches darauf abzielt, Kriege an der europäischen Peripherie führen und erfolgreich zu Ende bringen zu können (einschließlich mit durch Nuklearwaffen untermauerter Eskalationsdominanz). Da die NATO nicht beabsichtigt und auch nicht plant, einen Krieg gegen Russland aus dessen Peripherie heraus zu führen, müssen derartige Vorbereitungen auf russischer Seite als Versuch gesehen werden, für von Moskau initiierte regional begrenzte Kriege im baltischen Raum oder in der Schwarzmeerregion die Bedingungen dafür zu schaffen, dass die NATO nicht eingreift und so die Abschreckung der NATO unterlaufen werden kann. Diese Anstrengungen im Bereich der Mittelstreckenwaffen passen in das Muster einer russischen Politik, welche die strategische Konfrontation mit dem Westen sucht und die internationale, von westlichen Vorstellungen (insbesondere von der Bedeu-

23 NATO Brussels Summit Declaration 2018; Brauß/Mölling 2019, S. $3 / 4$ tung von Rüstungskontrolle) geprägte Ordnung zerstören möchte.

Für deutsche wie europäische Sicherheit bedeutet diese Erkenntnis, dass die Konzepte von Abschreckung und Rüstungskontrolle angepasst werden müssen, denn die Lage hat sich mit der Einführung russischer Mittelstreckenwaffen qualitativ verändert und wird sich weiter ändern.

Was die Rolle von Abschreckung betrifft, so ist es in erster Linie geboten, die Fähigkeiten zur Abwehr konventioneller oder hybrider Aggressionen im Baltikum und in Ostpolen so zu verstärken, dass sich Russland keine Chancen auf ein rasches fait accompli in dieser Region ausrechnen kann. ${ }^{24}$ In diesem Zusammenhang ist es von besonderer Bedeutung, dass die von der NATO beschlossenen Maßnahmen zur Erhöhung der Reaktionsfähigkeit und der Einsatzbereitschaft ihrer schnellen Reaktionskräfte und ihrer weiteren Verstärkungskräfte konsequent und so rasch wie möglich umgesetzt werden. Sie müssen eingebettet sein in eine kohärente Strategie und gründliche Vorausplanung. Die NATO-Militärbehörden entwickeln daher derzeit eine NATO-Militärstrategie für Abschreckung und Verteidigung in Europa und den angrenzenden Seegebieten. Sie wird die erste seit 1967 sein, als mit der legendären MC 14/3 die Strategie der Flexible Response etabliert wurde.

Deutschland kommt bei alledem eine Schlüsselrolle zu. Aufgrund seiner zentralen Lage hätte die Bundeswehr die Aufgabe, zusammen mit den USA bedrohte oder angegriffene Verbündete mit als erste zu verstärken. Als zentraleuropäische Drehscheibe hätte Deutschland die Verlegung nordamerikanischer und (west-)europäischer Streitkräfte zu ermöglichen und deren Aufmarsch zu schützen. Die Bundeswehr muss dazu so rasch wie möglich die volle Einsatzbereitschaft erreichen und anspruchsvolle zusätzliche Streitkräfteziele der NATO erfüllen. Die Verteidigungsministerin hat besondere und bisher erfolgreiche Anstrengungen unternommen, um zusammen mit zahlreichen kontinentaleuropäischen Verbündeten unter deutscher Federführung als Rahmennation militärische Fähigkeiten zu entwickeln und multinationale Verbände und Großverbände aufzustellen. ${ }^{25}$ Die Bundesregierung muss aber auch die notwendigen Mittel bereitstellen, um das erforderliche Fähigkeitsprofil der Bundeswehr zu finanzieren. Dies ist nur möglich, wenn die Bundesregierung den Wehretat Jahr für Jahr signifikant erhöht. Wie alle anderen

24 Vgl. zur Rolle von Abschreckung unter den heutigen Bedingungen De Wijk 2018 sowie Bergeron 2018.

25 Meyer zum Felde 2018, $114 \mathrm{f}$. 
Verbündeten hat sie sich verpflichtet, bis zum Jahre 2024 das Ziel zu erreichen, mindestens 2 Prozent des Bruttoinlandprodukts für Verteidigung auszugeben - was derzeit von Teilen der Bundesregierung befremdlicherweise in Frage gestellt wird.

Darüber hinaus wird in der NATO eine Neubewertung der nuklearen Fähigkeiten und Übungen notwendig werden. ${ }^{26}$ Diese muss dazu führen, dass die Glaubwürdigkeit der Abschreckungs- und Verteidigungsfähigkeit gegenüber dem wachsenden russischen Potential und angesichts der integrierten konventionell-nuklearen Militärdoktrin Moskaus erhalten bleibt. Sie muss dazu beitragen, dass diejenigen Waffensysteme beschafft werden, die am ehesten geeignet sind das russische Risikokalkül zu beeinflussen.

Was die Rolle von Rüstungskontrolle betrifft, so macht es nach dem russischen Vertragsbruch wenig Sinn, am INF-Vertrag festhalten zu wollen. Und selbst wenn es gelänge, Russland zum Abbau und zur kontrollierten Vernichtung der landgestützten 9M729-Marschflugkörper $\mathrm{zu}$ bewegen, bliebe die Bedrohung durch see- und luftgestützte Mittelstreckenwaffen bestehen. Unter diesem Problem litt der INF-Vertrag eigentlich von Beginn an. Aber da er eine gesamte Kategorie an nuklearen Waffen eliminierte und außerdem bei Vertragsabschluss (1987) absehbar war, dass dieser ein wichtiger Schritt in Richtung der Beendigung des Ost-West-Konflikts darstellen könnte, hoffte man auf weitere Fortschritte in der Rüstungskontrolle und Abrüstung. Schließlich wurde mit dem Ende des politischen Konflikts und der Auflösung des Warschauer Pakts und dann der Sowjetunion wie auch dem Niedergang ihrer Streitkräfte auch deren Invasionsfähigkeit beseitigt, und damit erledigte sich das Problem einer sowjetischen/russischen Eskalationsdominanz. In den folgenden Jahren ging man bei der NATO davon aus, dass Russland rund zehn Jahre benötigen würde, um die Fähigkeit zu einem großangelegten Angriff wiederzuerlangen. Außerdem waren seinerzeit die see- und luftgestützten sowjetischen Mittelstrecken-Marschflugkörper weniger leistungsfähig und flexibel als die landgestützten ballistischen Raketen und Marschflugkörper. Heute ist zum einen die politische Lage anders, weil Moskau nicht die Verständigung mit dem Westen sucht, und zum anderen sind die Leistungsmerkmale der russischen see- und landgestützten Marschflugkörper verbessert worden. Zwischen 2015 und 2018 kam es immer wieder zu relativ punktgenauen Angriffen see- und U-Boot-gestützter Kalibr-Flugkörper auf Stellungen der Terrororganisation Islamischer Staat in

26 Kamp 2017.
Syrien und der dortigen Opposition. Das war sicher auch ein Signal an den Westen. Derartiges könnte sich auch in Europa wiederholen.

Auch ein globalisierter INF-Vertrag, dem neben den USA und Russland andere Staaten wie China, Indien oder Pakistan beitreten könnten, erscheint auf absehbare Zeit politisch völlig unrealistisch, wenn man deren sicherheitspolitische Lage und ihre strategischen Interessen in ihren Regionen realistisch in Rechnung stellt. Ein globaler Verbotsvertrag für alle Mittelstrecken (land-, see- und luftgestützt) wäre noch weniger aussichtsreich.

Wenn ein Interesse daran besteht, in der heutigen Lage Rüstungskontrolle zu reaktivieren, dann muss diese völlig neu angedacht werden. Sie muss diejenigen Aspekte der Bedrohungslage ansprechen, die für unsere Sicherheit und die unserer Verbündeten am meisten relevant sind, beispielsweise das Wiederentstehen einer Invasionsfähigkeit Russlands, wenngleich regional begrenzt, sowie das damit verbundene Streben Russlands nach nuklearer Eskalationsdominanz. Ebenso muss sie Ansatzpunkte dafür finden, wo ein Interesse Russlands besteht, sich aus eigenem Sicherheitsinteresse auf ernsthafte Rüstungskontrollverhandlungen einzulassen. Lediglich Gespräche im Rahmen einer strukturierten Agenda zu führen - wie derzeit im Rahmen der OSZE - dürfte nicht ausreichen. Entscheidend ist es, Hebel zu finden, die Russland dazu bewegen können, seine generelle Politik gegenüber dem Westen zu ändern. Eine derartige Strategie ist derzeit weder im westlichen Bündnis noch in Berlin zu erkennen.

\section{Literatur}

Adamsky, Dmitry (2015): Cross-Domain Coercion. The Current Russian Art of Strategy. Paris: IFRI.

Bergeron, James Henry (2018): Die Dynamik der Abschreckung, SIRIUS, 2 (1), 21-32.

Brauß, Heinrich (2018): NATO Beyond 70: Renewing a Culture of Readiness. Tallinn: International Centre for Defence and Security. Vershbow, Alexander R. / Breedlove, Philip M. u. a. (2019): Permanent Deterrence. Enhancements to the US Military Presence in North Central Europe. Washington, D.C.: The Atlantic Council, Scowcroft Center for Strategy and Security. Brauß, Heinrich/Mölling, Christian (2019): Europas Sicherheit ohne INF-Vertrag. Politische und strategische Handlungsoptionen für Deutschland und die NATO. Berlin: Deutsche Gesellschaft für Auswärtige Politik (DGAP Kompakt).

Covington, Stephen R. (2016): The Culture of Strategic Thought Behind Russia's Modern Approaches to Warfare. Cambridge, Mass: Harvard University, Belfer Center.

Department of Defense (2018): Nuclear Posture Review 2018. Washington, D.C: DoD. 
De Wijk, Rob (2018): Die Rolle von Abschreckung im neuen strategischen Umfeld Europas, SIRIUS, 2 (1), 3-20.

Dick, Charles (2019): Russian Ground Forces Posture Towards the West. London: Chatham House (Research Paper).

Giles, Keir (2016): Russia's New Tools for Confronting the West. Continuity and Innovation in Moscow's Exercise of Power. London: Royal Institute of Internationale Affairs.

Hedlund, Stefan (2017): Aurora 2017 - ein neuer Beginn für Schwedens Verteidigung, und für die NATO, SIRIUS, 1 (4), 389-390.

Johnson, Dave (2018): Russia's Conventional Precision Strike Capabilities, Regional Crises, and Nuclear Thresholds. Livermore, Cal.: Lawrence Livermore National Laboratory, Center for Global Security Research.

Johnson, Dave (2019): General Gerasimov on the Vectors of the Development of Military Strategy. Rom: NATO Defence College.

Kamp, Karl-Heinz (2017): Eine neue Nuklearausrichtung der NATO, SIRIUS, 1 (4), 359-366.

Kramer, Franklin/Binnendijk, Hans (2018): Meeting the Russian Conventional Challenge. Effective Deterrence by Prompt Reinforcement. Washington, D.C.: Atlantic Council.
Kristensen, Hans M. (2018): Russland modernisiert Kernwaffendepot im Bezirk Kaliningrad, SIRIUS, 2 (4), 398-400.

Kroenig, Matthew (2018): Russlands Nuklearstrategie gegenüber Europa. Wie organisiert man Abschreckung gegen Deeskalation mit nuklearen Schlägen?, SIRIUS, 2 (4), 323-338.

Meyer zum Felde, Rainer (2018): Abschreckung und Dialogbereitschaft. Der Paradigmenwechsel der NATO seit 2014, SIRIUS, 2 (2), 101-117.

Nilsen, Thomas (2018): Russland erweitert im großen Umfang seine Kernwaffenlager auf der Kola-Halbinsel, SIRIUS, 2 (4), 401-405.

Office of the US Secretary of Defence (2018): Nuclear Posture Review, February 2018, Washington D.C.

Palmer, Diego Ruiz (2018): Theatre Operations, High Commands and Large-Scale Exercises in Soviet and Russian Military Practice. Insights and Implications. Rom: NATO Defence College.

Peters, Robert/Anderson, Justin/Menke, Harrison (2018): Deterrence in the 21st Century: Integrating Nuclear and Conventional Force, Strategic Studies Quarterly, 12 (4), 15-43.

Warden, John K. (2018): Limited Nuclear War. The 21 ${ }^{\text {st }}$ Century Challenge for the United States. Livermore, Cal.: Lawrence Livermore National Laboratory, Center for Global Security Research.

\section{Anhang: Nuklearfähige russische Marschflugkörper im Mittelstreckenbereich}

\begin{tabular}{|c|c|c|c|c|}
\hline Name & Plattform & Reichweite & $\begin{array}{l}\text { Indienst- } \\
\text { stellung }\end{array}$ & Anmerkungen \\
\hline $9 M 729$ & Landgestützt, mobil & $2.000 \mathrm{~km}+$ & (2017?) & $\begin{array}{l}\text { Derzeit } 4 \text { Bataillone mit ins- } \\
\text { gesamt } 64 \text { Werfern }\end{array}$ \\
\hline 3M14 T/S Kalibr NK & $\begin{array}{l}\text { Überwasserschiffe, R314 } \\
\text { und R314K Werfer; }\end{array}$ & $1.600-2.600 \mathrm{~km}$ & 2011 & $\begin{array}{l}\text { Die russische Marine rüstet } \\
\text { derzeit mehrere Schiffstypen } \\
\text { unterschiedlicher Größe mit } \\
\text { diesen FK aus }\end{array}$ \\
\hline $\begin{array}{l}\text { 3M14 PL, Kalibr (NATO } \\
\text { Code: SS-N-30A }\end{array}$ & $\begin{array}{l}\text { U-Boote der Yasen Klassen, } \\
\text { sowie auch der Akula Klasse }\end{array}$ & $1.500-2.500 \mathrm{~km}$ & 2017 & $\begin{array}{l}1 \text { Boot der Yasen Klasse in } \\
\text { Dienst, } 7 \text { weitere im Zulauf oder } \\
\text { im Bau }\end{array}$ \\
\hline $\begin{array}{l}\text { RK-55 (NATO Code: SS-10 } \\
\text { Grant) }\end{array}$ & U-Boote der Akula Klasse & $2.400 \mathrm{~km}$ & 1986 & $\begin{array}{l}\text { Es sollen noch } 150 \text { bis } 180 \\
\text { Raketen verfügbar sein }\end{array}$ \\
\hline $\begin{array}{l}\text { Kh-55 (NATO Code: AS-15 } \\
\text { Kent) }\end{array}$ & $\begin{array}{l}\text { Luftgestützt, Tu-95MS, } \\
\text { Tu-142M, Tu-160; Tu22M3, } \\
\text { Tu-22 }\end{array}$ & $2.500 \mathrm{~km}$ & 1984 & \\
\hline Kh-55SM & $\begin{array}{l}\text { Luftgestützt, Tu-95MS, } \\
\text { Tu-142M, Tu-160; Tu22M3, } \\
\text { Tu-22; }\end{array}$ & $3.000 \mathrm{~km}$ & 1987 & \\
\hline Kh-101/102 & $\begin{array}{l}\text { Tu95MS, Tu-160, Tu-M3; } \\
\text { Su-34 }\end{array}$ & $2.200-5.500$ & 2013 & $\begin{array}{l}\text { Die Kh } 101 \text { ist die konven- } \\
\text { tionelle, die Kh } 102 \text { die } \\
\text { nukleare Variante }\end{array}$ \\
\hline Kh-47M2 Kinzhal & Mig-31B & $2.000 \mathrm{~km}+$ & & Erfolgreich getestet \\
\hline
\end{tabular}

Ohne Marschflugkörper, die als Anti-Schiffswaffen konzipiert sind 